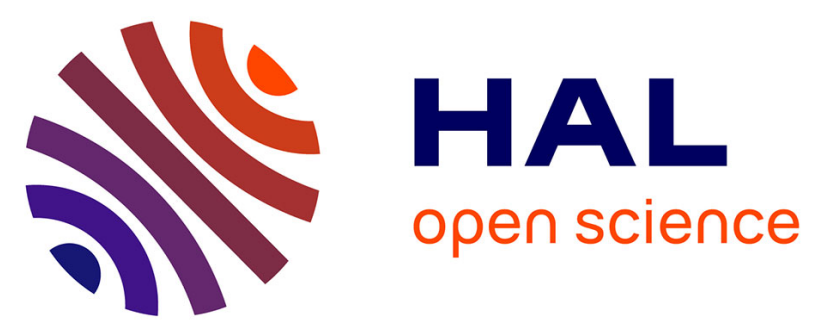

\title{
Behavioural templates improve robot motion planning with social force model in human environments
}

Alessio Colombo, Daniele Fontanelli, Dhaval Gandhi, Antonella de Angeli, Luigi Palopoli, Sean Sedwards, Axel Legay

\section{- To cite this version:}

Alessio Colombo, Daniele Fontanelli, Dhaval Gandhi, Antonella de Angeli, Luigi Palopoli, et al.. Behavioural templates improve robot motion planning with social force model in human environments. 2013 IEEE 18th Conference on Emerging Technologies

Factory Automation (ETFA), Sep 2013, Cagliari, Italy. pp.1 - 6, 10.1109/ETFA.2013.6648081 . hal01088063

\section{HAL Id: hal-01088063 \\ https://hal.inria.fr/hal-01088063}

Submitted on 27 Nov 2014

HAL is a multi-disciplinary open access archive for the deposit and dissemination of scientific research documents, whether they are published or not. The documents may come from teaching and research institutions in France or abroad, or from public or private research centers.
L'archive ouverte pluridisciplinaire HAL, est destinée au dépôt et à la diffusion de documents scientifiques de niveau recherche, publiés ou non, émanant des établissements d'enseignement et de recherche français ou étrangers, des laboratoires publics ou privés. 


\title{
Behavioural Templates Improve Robot Motion Planning with Social Force Model in Human Environments*
}

\author{
Alessio Colombo, Daniele Fontanelli, Dhaval Gandhi, Antonella De Angeli, Luigi Palopoli \\ University of Trento, Trento, Italy. \\ Sean Sedwards, Axel Legay \\ Inria Rennes - Bretagne Atlantique, Rennes, France.
}

\begin{abstract}
An accurate model of human behaviour is crucial when planning robot motion in human environments. The Social Force Model (SFM) is such a model, having parameters that control both deterministic and stochastic elements. We have constructed an efficient motion planning algorithm by embedding the SFM in a control loop that determines higher level objectives and reacts to environmental changes. Low level predictive modelling is provided by the SFM fed by sensors; high level logic is provided by statistical model checking.

To parametrise and improve our motion planning algorithm, we have conducted experiments to consider typical human interactions in crowded environments. We have identified a number of behavioural patterns which may be explicitly incorporated in the SFM to enhance its predictive power. In this paper we describe the results of these experiments and how we parametrise the SFM.
\end{abstract}

\section{Introduction}

Many new applications for robots require them to operate in the proximity of human beings. Examples include robots that execute daily activities in homes or public spaces (e.g., automatic cleaners), robots used to assist older adults or disabled people and a new generation of toys. In contrast to the traditional notion of industrial robots, whose areas of operation must be confined to prevent accidents [2], these new devices are autonomous and may interact with humans. In this context, the ability to understand human behaviour and social interactions is of primary importance.

Our research is motivated by the activities of the DALi project [1], whose goal is to develop an autonomous device to assist those with reduced ability. In particular, we are developing a 'cognitive engine' (an intelligent motion planner) to help the user reach places of interest in crowded public spaces, such as shopping malls, airports and railway stations. This can be challenging and stressful for those with reduced mental or physical ability.

Our motion planner employs a dynamic model of the local environment to hypothesise trajectories of moving

${ }^{*}$ This work is partially funded by the Devices for Assisted Living (DALi) European project, reference 288917. [1]

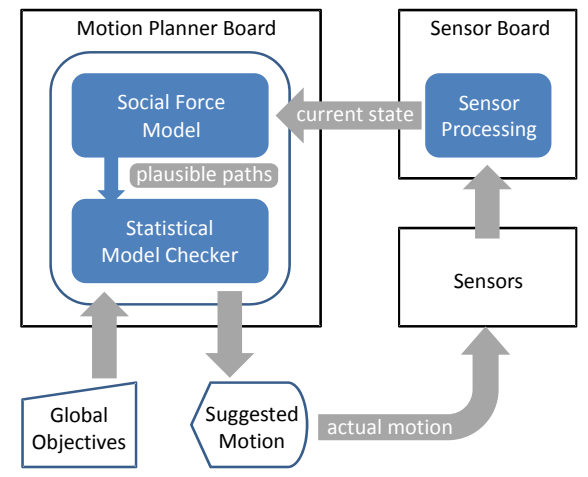

Figure 1. The DALi motion planner.

objects. For a given set of alternative actions, these predictions are analysed by higher level logic to decide the user's best course. The predictive model is based on the Social Force Model (SFM) [7, 6], using knowledge of prior trajectories and current sensor information. The control loop employs statistical model checking, with the user's objectives and constraints encoded in temporal logic [3]. An overview of the motion planner is shown in Fig. 1.

The SFM assumes that human motion respects the physical laws of motion and social conventions that may be modelled as forces. The SFM considers fixed objects (e.g., walls), moving objects (e.g., pedestrians), attractive forces (e.g., between friends or towards points of interest), repulsive forces (e.g., between strangers or away from crowds), frictional forces (when objects collide) and random terms that model uncertainty (unpredictable behaviour). There are alternative models of pedestrian dynamics (e.g., [18]), however the SFM suits our needs: it is flexible, efficient and is widely adopted in the literature (e.g., [7, 6, 11]). Its strengths and weaknesses are well tested and understood [10, 16, 13]. A mathematical description of the SFM is given in Section 2.

We and our collaborators ${ }^{1}$ have built prototypes to demonstrate the proof of concept of the scheme shown in Fig. 1. We have verified the efficiency of the algorithm on embedded computing hardware and confirmed that the mobile sensor technology can provide the required information. In this paper we focus on improving the social force model. Our goal is to increase its predictive accu-

${ }^{1}$ http://www.ics.forth.gr/cvrl 
racy and reduce the need for reactive corrections.

\subsection{The motion planning challenge}

The SFM is a model of continuous interaction (mutual reaction), driven by the desired trajectories of moving agents (denoted $\mathbf{v}^{0}(t)$, see Section 2$)$. When using the SFM in a generative context (e.g., [6]), $\mathbf{v}^{0}(t)$ may be specified in advance. The desired trajectories of real pedestrians, however, are a function of their objectives and the instantaneous positions and velocities of other pedestrians. In our motion planning application we are able to measure such positions and velocities, but only the objectives of the user are known with any certainty. The intentions of other pedestrians must be inferred from their trajectories. Noting that humans tend to walk in straight lines, we approximate the desired trajectories of other pedestrians piecewise, using short term linear extrapolations of their most recently detected motion. This is sufficient when the motion is smooth and the prediction timescale is short. In reality, the relatively smooth long and medium term trajectories of pedestrians are often interrupted by sudden short term pauses and deviations.

To improve the performance of the SFM, we have therefore investigated in detail the behaviour of human participants in a simulated shopping environment. We have defined an experimental procedure comprising a concurrent verbal protocol (to identify the motivation of the choices made by the participants), video recording and motion tracking using the Kinect platform [12]. The reconstructed trajectories of the participants were manually annotated with corresponding motivations, thus identifying a set of behavioural patterns. The data corresponding to each pattern were then used to parametrise the SFM using standard algorithms.

The results of our investigation show that, for some patterns, parametrisation of our existing model is sufficient to produce a good reconstruction of observed behaviours. In other cases, we find that it will be necessary to incorporate the patterns as explicit modifications to the SFM. Since the patterns have recognisable signatures, we conclude that we can construct an improved SFM using behavioural templates.

\section{The social force model}

Following [5], our model is constructed in two dimensions, with human agents represented by circular discs and fixed objects represented by lines. In what follows we denote vectors in bold type. Thus, agent $i$ has mass $m_{i}$ centered at position $\mathbf{x}_{i} \in \mathbb{R}^{2}$ in the environment, radius $r_{i}$ and velocity $\mathbf{v}_{i} \in \mathbb{R}^{2}$. The SFM is described by a system of linear differential equations

$$
\left\{\begin{array}{l}
\dot{\mathbf{x}}_{i}=\mathbf{v}_{i} \\
\dot{\mathbf{v}}_{i}=\frac{\mathbf{v}_{i}^{0}-\mathbf{v}_{i}}{\tau_{i}}+\frac{\mathbf{f}_{i}+\boldsymbol{\xi}_{i}}{m_{i}}
\end{array}\right.
$$

$\mathbf{v}_{i}^{0}$ is the driving (desired) velocity of agent $i$, represented by a product of speed $v_{i}^{0}$ and normalised direction $\mathbf{e}_{i}^{0}$. In our algorithm $\mathbf{e}_{i}^{0}$ is given by the line joining the current position and the next via point. Importantly, since $v_{i}^{0}$ is by default set to the user's preferred walking speed, $\mathbf{v}_{i}^{0}$ is time invariant between via points. $\tau_{i}$ is the time taken to react to the difference between desired and actual velocity, while $\boldsymbol{\xi}_{i}$ is a noise term modelling fluctuations not accounted for by the deterministic part of the model. The noise term can also serve to avoid deadlocks and hypothesise alternative trajectories. In our implementation we assume $\boldsymbol{\xi}_{i}$ is normally distributed. In the absence of the exogenous inputs $\mathbf{f}_{i}$ and $\boldsymbol{\xi}_{i}$, the agent's trajectory simply converges to the driving velocity with time constant $\tau_{i} . \mathbf{f}_{i}$ is the overall force acting on agent $i$ resulting from other objects in the environment and is given by

$$
\mathbf{f}_{i}=\sum_{j \neq i}\left[\mathbf{f}_{i j}^{\mathrm{soc}}+\mathbf{f}_{i j}^{\mathrm{att}}+\mathbf{f}_{i j}^{\mathrm{ph}}\right]+\sum_{b}\left[\mathbf{f}_{i b}^{\mathrm{soc}}+\mathbf{f}_{i b}^{\mathrm{ph}}\right]+\sum_{c} \mathbf{f}_{i c}^{\mathrm{att}}
$$

The first term on the right-hand side of (2) includes all the forces on agent $i$ resulting from interactions with other agents: $\mathbf{f}_{i j}^{\text {soc }}$ is the repulsive social force that inhibits strangers from getting too close, $\mathbf{f}_{i j}^{\text {att }}$ is the attractive social force that, e.g., brings friends together, $\mathbf{f}_{i j}^{\mathrm{ph}}$ is the physical force that exists when two people come into contact. The second term includes the forces acting on agent $i$ as a result of fixed environmental obstacles (e.g., walls): $\mathbf{f}_{i b}^{\text {soc }}$ is the social force that inhibits agent $i$ from getting too close to the boundaries, $\mathbf{f}_{i b}^{\mathrm{ph}}$ is the physical force that exists when agent $i$ touches the boundary $b$. Finally, $\mathbf{f}_{i c}^{\text {att }}$ is the attractive social force that draws agent $i$ towards fixed objects of incidental interest (shops, cafés, toilets, etc.).

$\mathrm{f}$ is principally a function of the distance between an agent and the other objects in the model. $d_{i b}$ is the minimum distance between the circumference of agent $i$ and fixed object $b . d_{i j}$ is the distance between the centres of mass of agents $i$ and $j$, i.e., the centres of the discs, while $r_{i j}=r_{i}+r_{j}$ is the "touching distance". To aid modelling the different force regimes that exist when agents are not in contact and when they touch (i.e. agents $i$ and $j$ touch if $\left.r_{i j}-d_{i j} \leq 0\right)$ we adopt the function $\Theta\left(r_{i j}, d_{i j}\right)=\max \left(0, r_{i j}-d_{i j}\right)$.

Using these notions, the various repulsive social and physical forces of (2)) are defined as follows:

$$
\begin{aligned}
\mathbf{f}_{i j}^{\text {soc }} & =\left\{A_{i} \exp \left[\left(r_{i j}-d_{i j}\right) / B_{i}\right]\right\} \mathbf{n}_{i j} \Lambda\left(\lambda_{i}, \varphi_{i j}\right) \\
\mathbf{f}_{i j}^{\mathrm{ph}} & =k_{1} \Theta\left(r_{i j}-d_{i j}\right) \mathbf{n}_{i j}+k_{2} \Theta\left(r_{i j}-d_{i j}\right) \Delta v_{j i}^{t} \mathbf{t}_{i j} \\
\mathbf{f}_{i b}^{\mathrm{soc}} & =\left\{A_{i} \exp \left[\left(r_{i}-d_{i b}\right) / B_{i}\right]+k_{1} \Theta\left(r_{i}-d_{i b}\right)\right\} \mathbf{n}_{i b} \\
\mathbf{f}_{i b}^{\mathrm{ph}} & =-k_{2} \Theta\left(r_{i}-d_{i b}\right)\left(\mathbf{v}_{i} \cdot \mathbf{t}_{i b}\right) \mathbf{t}_{i b}
\end{aligned}
$$

$\mathbf{n}_{i j}\left(\mathbf{n}_{i b}\right)$ is a normalised vector pointing from agent $j$ (fixed object $b$ ) to agent $i$, i.e., the direction of the repulsive force. $\mathbf{t}_{i j}\left(\mathbf{t}_{i b}\right)$ is a normalised vector tangential to the relative movement of agent $i$ and agent $j$ (fixed obstacle $b$ ), i.e., the motion tangential direction. $\Delta v_{j i}^{t}=$ $\left(\mathbf{v}_{j}-\mathbf{v}_{i}\right) \cdot \mathbf{t}_{i j}$ is the tangential velocity difference. The social forces (3) and (5) increase exponentially with reducing distance between objects, with a scale defined by constants $A_{i}$ and $B_{i}$. In particular, $A_{i}$ is the force acting on agent $i$ at the touching distance; $B_{i}$ is loosely the distance at which the force takes effect.

$\Lambda: \mathbb{R}^{2} \mapsto[0,1]$ is a function that gives greater weight to the social force (3) arising from the agents in front of (notionally, seen by) an agent. $\lambda_{i}$ is a parameter that regulates the effect of $\Lambda$ on agent $i$, while $\varphi_{i j}$ is the angle 


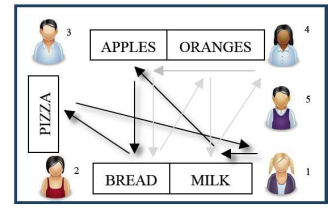

(a) Diagrammatic scheme of participants and shops showing potential trajectories

Figure 2. Experimental set-up.

between the directions $\mathbf{e}_{i}^{0}$ and $-\mathbf{n}_{i j}$, i.e., the field of view of the agent. The physical force (4) between agents comprises a repulsive body compression force (first term) that acts in direction $\mathbf{n}_{i j}$, plus a frictional force (second term) that acts in direction $\mathbf{t}_{i j}$ to impede the relative tangential movement of two agents in contact. $k_{1}$ and $k_{2}$ are constants that define the scale of the physical forces. The physical force (6) between an agent and a fixed object is solely described by a frictional term.

\section{Qualitative studies}

A qualitative user study was conducted to collect information about how people behave in crowded environments. We considered three main themes: (i) how people interact in confined environments; (ii) how people negotiate shared space and (iii) how people behave with other people in shared space. A simplified shopping context was constructed in a laboratory, as illustrated in Fig. 2. Our experimental procedure involved the use of a concurrent protocol, video-recorded observation and questionnaire administration. These techniques have been found to be particularly effective when conducting experimental investigations $[8,14]$. The concurrent or talk-aloud protocol - a narration of thought and action during action - was chosen as literature suggests the alternative retrospective protocol (where participants return to view and comment upon their recorded experience) may not accurately reveal participants actual task performance experience. Concurrent protocol participants have been found to go into greater detail and provide more in-depth evaluations [15].

\subsection{Procedure}

The participant sample consisted of 25 University students. Five participants at a time were involved in each experiment, and assigned a different list of four shopping items to collect. They were asked to collect all items in the shortest time possible, verbally explaining all their actions. Participants were specifically arranged around the shopping environment and each of the five shopping lists were unique, to maximise shared space interaction and limit the possibility of processional behaviour. The potential route-behaviour of participants 1 and 4 is illustrated in Fig. 2(a). Participants were asked to collect the items on their list and return to their starting points, while simultaneously verbalising their shopping experience; describing what they saw, where they went, and what they were thinking and doing. The voice-recording functionality of five HUAWEI U8650 Android mobile phones was utilised to capture the speech of the participants. Three Logitech Quickcam Pro 9000 webcams were used to observe and record shared space interaction within the simulated shopping environment. The cameras were coordinated to provide a more encompassing view of the interaction space (Fig. 2(b)). On completion of the experiment, the participants were asked to complete an open questionnaire.

\subsection{Data analysis}

The transcribed concurrent protocols, once synchronized with the video footage and in conjunction with the development of relevant coding schemes, facilitated assessment of a number of variables, including Task Completion Time, Number of Steps taken, and Number of Critical Instances - instances where physical, visual and/or auditory reference was made within the verbal protocols to agent-agent interaction. Detailed video analysis was conducted via the Elan software tool [17].

\subsection{Results}

Using the Critical Instances markers from the transcribed protocols synchronised to the video footage, we documented and studied the behaviour exhibited by participants when involved in shared space interaction. Critical instances were defined as physical, visual and/or auditory references within the verbal protocols to agent-agent awareness or interaction. Analysis of the identified critical instances revealed a number of common themes and behaviours. The themes that emerged indicated that the behaviour itself was usually employed to either negotiate shared space interaction or avoid collisions within the shared space, and it was possible to categorise the main types of behaviours in two groups: Active and Reactive behaviours.

Active behaviours, in this instance, were considered to be behaviours employed to understand the environment and determine goal strategies toward task completion, environmental awareness and negotiation, mainly focussing around the visual modality:

(A) Eye-to-eye negotiation of immediate shared space;

(B) Use of peripheral vision in assessment;

(C) Visual scanning of environment;

(D) Verbal interaction.

Reactive behaviours were considered as the reactions of agents in the environment to other agents; the physical reactive movements made to accommodate other agents and successful interaction:

(E) Waiting for space/desired location to become clear;

(F) Stepping backwards to allow others more room;

(G) Moving forwards to allow others more room;

(H) Stationary agent yielding to moving agent;

(I) Move left;

(J) Move right.

Table 1 provides an example of how the critical instances were interpreted in terms of the taxonomy of behaviours observed during shared space interaction. By examining critical instances within the complete video footage, it was possible to observe the interactional behaviour occurring during such periods according to the 


\begin{tabular}{|c|l|}
\hline Crit. Inst. & Observed Behaviour of Participant $(P x)$ \\
\hline 1 & Px aware of other agent in desired space, waits until agent completes task (behaviours (E), (H), (C)) \\
\hline 2 & Backward glance locates agent directly behind Px and helps avoid collision (behaviour (B)) \\
\hline 3 & $\begin{array}{l}\text { Px aware of an agent in front and another approaching agent (behaviours (B), (C)) } \\
\text { Px watches the actions of a moving agent and remains still until the agent passes (behaviours (E), (H)) }\end{array}$ \\
\hline
\end{tabular}

Table 1. Critical instances interpreted in terms of the taxonomy of behaviours.

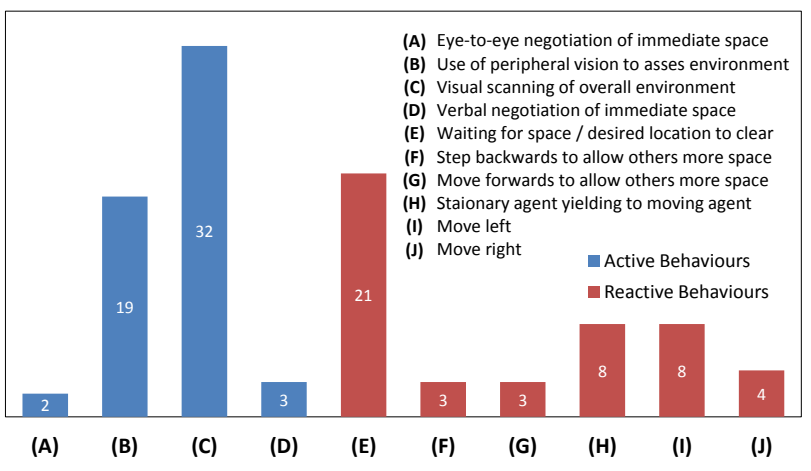

Figure 3. Distribution of behaviours.

\begin{tabular}{|c|c|c|l|}
\hline Rule & Agent 1 & Agent 2 & Behaviour \\
\hline 1 & Active & Passive & Agent 2 steps back and allows agent 1 \\
\hline 2 & Active & Passive & Agent 1 moves and shares space \\
\hline 3 & Active & Passive & Agent 1 waits for empty space \\
\hline 4 & Active & Active & Agent 1 moves left or agent 2 moves right \\
\hline 5 & Active & Active & Agent 1 waits and gives way to agent 2 \\
\hline
\end{tabular}

Table 2. Identified behavioural rules.

taxonomy, and to determine the frequency with which these behaviours occurred (Fig. 3). As can be observed in Fig. 3, participants utilised the visual modality to engage in either scanning of the overall environment (usually to identify product locations within it), or peripheral vision to monitor the orientational movement of other agents in the environment. As proximity to others in the environment increased, eye-to-eye contact or negotiation between participants was observed to occur. Similarly, under these circumstances, verbal negotiation was also evident in a small number of cases, although this may have been reduced due to the method of recording the concurrent protocols. The most commonly occurring reactive behaviours according to the study were waiting for free space to become available, stationary agents giving priority to moving agents, moving to the left and right, and moving forwards and backwards to create free space, based upon the interactions occurring during the themes defined.

From the analysis of the videos it was evident that people planned their movement based on their physical distance from others, broadly in line with the assumptions of the SFM. However, we observed modes of behaviour that are not explicitly modelled by the SFM. When somebody came too close to another person, avoidance behaviours were manifested, such as waiting (for the space to be free) or stepping back. These behaviours were clustered in 5 main rules, as reported in Table 2. It is also interesting to note that when two active agents meet, the slower one typically gives way to the faster.

Our study highlights the fundamental role of proxemics [4] in human motion. A literature exists concerning psy- chological aspects of spatial behaviour, taking into consideration concepts such as proximity, body orientation, motion in a physical setting, territorial behaviour and privacy. These concepts can provide a theoretical framework to extend the SFM, informing our understanding of people's behaviour in public spaces.

\section{Parametrising the SFM}

We conducted a number of motion tracking experiments using the simulated shopping environment described in Section 3. We performed 20 experiments considering two people in the environment and a further 20 experiments using four people. Participants were arranged in specific places around the shopping environment and asked to move according to a shopping list provided in advance. Each shopping list contained a set of places to be visited (via points) in a predefined order, to prompt interactions between participants and thus generate interesting social behaviours. Depending on the number of participants, shopping lists were specifically designed to maximise shared space interaction and limit the possibility of processional behaviour. We recorded video and 3D information of the participants' trajectories using the Kinect sensing apparatus [12].

\subsection{Parameter estimation}

Once the experimental data were collected, we decomposed the participants' trajectories according to the SFM, considering situations where there were at least two agents that interacted, having simultaneously crossing trajectories. Since each participant had been asked to travel through a sequence of via points and to pause in each starting and ending position, it was possible to identify the relevant segments of their trajectories in the experimental data. By assuming that the driving velocity of agents is constant along a line joining the initial and desired positions (see Section 2) we were able to infer $\mathbf{v}^{0}$.

Due to the constraint imposed by the initial and final configurations, for the purposes of parameter estimation we construct a modified version of the SFM described in Section 2. We first consider the original model (1) and define $\mathbf{x}_{i}^{0}=\left[x_{i}^{0}, y_{i}^{0}\right]^{T}$ and $\mathbf{v}_{i}^{0}=\left[v_{x_{i}}^{0}, v y_{i}{ }^{0}\right]^{T}=[0,0]^{T}$ to be the desired final position and velocity, respectively, of agent $i$. We thus define the Cartesian position error variables $\tilde{\mathbf{x}}_{i}=\mathbf{x}_{i}^{0}-\mathbf{x}_{i}$, whose dynamics are given by $\dot{\tilde{\mathbf{x}}}_{\mathbf{i}}=-\mathbf{v}_{\mathbf{i}}$. Furthermore, we define the polar coordinates

$$
\rho_{i}=\sqrt{\tilde{\mathbf{x}}_{i}^{T} \tilde{\mathbf{x}}_{i}}, \quad \alpha_{i}=\arctan \left(\frac{\tilde{y}_{i}}{\tilde{x}_{i}}\right)
$$

which are respectively the distance and the orientation from the current to the desired position of agent $i$. We 
then find that the normalised direction $\mathbf{e}_{i}^{0}$ turns out to be

$$
\mathbf{e}_{i}^{0}=\left[\begin{array}{c}
e_{x_{i}}^{0} \\
e_{y_{i}}^{0}
\end{array}\right]=\left[\begin{array}{c}
\frac{\tilde{x}_{i}}{\sqrt{\tilde{\mathbf{x}}_{i}^{T} \tilde{\mathbf{x}}_{i}}} \\
\frac{\tilde{y}_{i}}{\sqrt{\tilde{\mathbf{x}}_{i}^{T} \tilde{\mathbf{x}}_{i}}}
\end{array}\right]=\left[\begin{array}{c}
c_{\alpha_{i}} \\
s_{\alpha_{i}}
\end{array}\right],
$$

where we adopt the convention $c_{\alpha_{i}}=\cos \left(\alpha_{i}\right)$ and $s_{\alpha_{i}}=$ $\sin \left(\alpha_{i}\right)$. Hence, the modified SFM is finally given by the dynamical system

$$
\left[\begin{array}{c}
\dot{\rho}_{i} \\
\dot{\alpha}_{i} \\
\dot{v}_{x_{i}} \\
\dot{v}_{y_{i}}
\end{array}\right]=\left[\begin{array}{c}
-\left(c_{\alpha_{i}} v_{x_{i}}+s_{\alpha_{i}} v_{y_{i}}\right) \\
\frac{s_{\alpha_{i}} v_{x_{i}}-c_{\alpha_{i}} v_{y_{i}}}{\rho_{i}} \\
\frac{v_{i}^{0} c_{\alpha_{i}}-v_{x_{i}}}{\tau_{i}}+\frac{f_{x_{i}}+\xi_{x_{i}}}{m_{i}} \\
\frac{v_{i}^{0} s_{\alpha_{i}}-v_{y_{i}}}{\tau_{i}}+\frac{f_{y_{i}}+\xi_{y_{i}}}{m_{i}}
\end{array}\right] .
$$

Using (7), (8) and (9) it is then possible to have an approximate description of the participant accelerations, i.e.,

$$
\frac{\mathbf{v}_{i}\left(t_{k+1}\right)-\mathbf{v}_{i}\left(t_{k}\right)}{t_{k+1}-t_{k}} \approx \frac{\mathbf{g}_{i}\left(t_{k}\right)+\mathbf{g}_{i}\left(t_{k+1}\right)}{2}
$$

where

$$
\mathbf{g}_{i}\left(t_{k}\right)=\frac{v_{i}^{0} \mathbf{e}_{i}^{0}\left(t_{k}\right)-\mathbf{v}_{i}\left(t_{k}\right)}{\tau_{i}}+\frac{\mathbf{f}_{i}\left(t_{k}\right)+\boldsymbol{\xi}_{i}\left(t_{k}\right)}{m_{i}} .
$$

Note that calculating the mean of successive values of $\mathbf{g}_{i}(\cdot)$ gives a constant mean acceleration in the sampling period $t_{k+1}-t_{k}$.

Having set the desired speed of each agent to $v_{i}^{0}=$ $\max \left\{\left\|\mathbf{v}_{i}\left(t_{k}\right)\right\|\right\}$, we estimated values for parameters $A_{i}$ and $B_{i}$ in (3) and (5), and for parameter $\tau_{i}$ in (1). We note here here that in our experiments the agents never touch, so the constants $k_{1}$ and $k_{2}$ are not used and are not estimated. Moreover, the $\Lambda(\cdot)$ function is for the moment assumed to be equal to 1 , without loss of generality, since the trajectories do not take into account interactions from behind. Finally, the mass of the participants $m_{i}$ plays only the role of a weighting factor for the generated forces, hence it is of no relevance to the problem at hand and can be considered as known in advance and removed form the estimation process.

\subsection{Estimation algorithm}

According to the description of the forces and the assumption that the model noise term $\boldsymbol{\xi}_{i}$ is normally distributed, we adopt an iterative Weighted Least Squares (WLS) algorithm to identify the parameters of the dynamical system. Such a choice is justified by the limited number of parameters involved in the estimation and by the relatively small amount of noise in determining the positions of the participants. This method is alternative to [9], in which a genetic algorithm based solely on video tracking data is used to estimate parameters. In our case, we make use of the high quality output of the Kinect sensor.

\subsection{Results}

Fig. 4 illustrates typical instantaneous output from an experiment: Fig. 4(a) shows a grabbed image of the simulated shopping environment; Fig. 4(b) shows a plan view of the corresponding tracked participants. Each participant is represented by a disk of radius $r_{i}$ - the same value

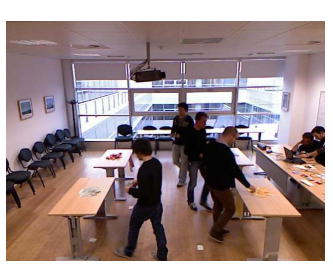

(a) Video grab.

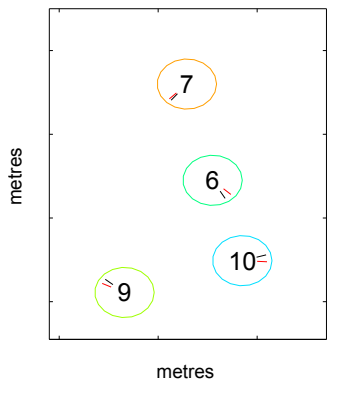

(b) Tracked participants.

\section{Figure 4. Instantaneous output of experi- ment.}

used in (3), (4), (5) and (6) - and identified by a unique number for tracking purposes.

Fig. 5 illustrates typical global trajectories, reconstructed from the tracked positions of the participants. The global trajectories were divided into local trajectories describing the motion between the pre-defined via points. Even without time information, the locations of the via points are clear in Fig. 5. The local trajectories were then used to estimate parameters of the SFM, to better "predict" the actual trajectories.

In Fig. 6(a) the local trajectories of two participants going in opposite directions are represented by solid lines. The dashed lines represent the trajectories generated by the parametrised SFM. The figure demonstrates the good agreement of the SFM with reality in situations where only relatively small corrections are necessary and there is little conflict.

In Fig. 6(b) the interaction is more conflictual and the negotiation of shared space increases in complexity. In this case our simple linear extrapolation to infer the participants' desired trajectories is not adequate and the SFM's prediction is poor. Interestingly, the point at which the actual and predicted trajectories diverge (identified by a circle in the figure) is the point at which one agent stops to give way to the other. We have found such active behaviour, i.e., moving to facilitate the motion of other agents or waiting for free space, to be most frequent in our experimental context (see Fig. 3).

\section{Conclusions and prospects}

Our unmodified social force model is accurate under conditions of continuous flow and when there is little competition for space. Under other circumstances, common in the social environments that are of interest to us, human motion is punctuated by frequent stops and starts, and short term changes of direction. Our experiments have demonstrated that these discrete events are not well captured by a simple social force model. We have nevertheless identified several distinguishable behavioural patterns that may be incorporated as templates into an enhanced SFM. In such a model, these patterns would be recognised and predicted, modifying the desired trajectories of modelled pedestrians. We have shown that this increases the 


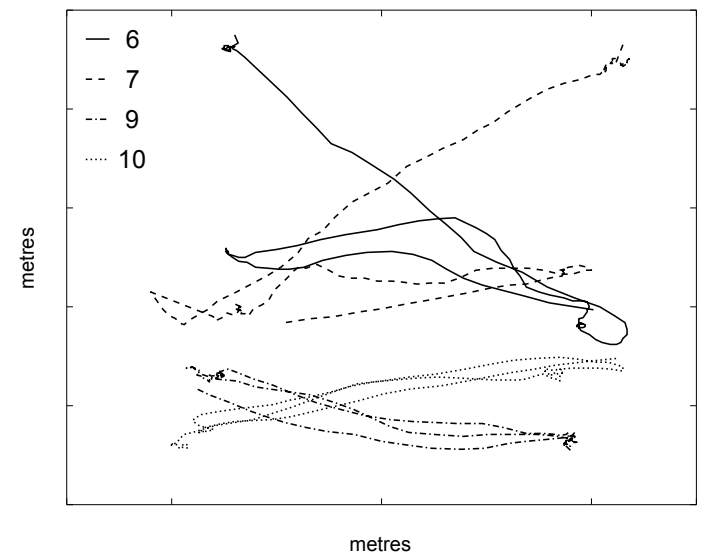

Figure 5. Trajectories corresponding to experiment shown in Fig. 4.

predictive accuracy of the SFM.

We note that the patterns we have identified might be explained by a more general theory of human interaction. We therefore propose to explore proxemics theory [4], which has been developed to explain behaviours of people when they interact in close proximity. We also recognise that our investigations thus far have considered only a limited range of social situations. Key to our ongoing research will be an extensive collection of experimental data that can inform our theory and provide evidence of its applicability and robustness.

\section{References}

[1] The DALi Project. http://www.ict-dali.eu/dali, November 2011.

[2] R. Asaula, D. Fontanelli, and L. Palopoli. Safety provisions for human/robot interactions using stochastic discrete abstractions. In Proc. of 2010 IEEE/RSJ International Conference on Intelligent Robots and Systems (IROS2010), October 2010.

[3] A. Colombo, D. Fontanelli, A. Legay, L. Palopoli, and S. Sedwards. Motion Planning in Crowds using Statistical Model Checking to Enhance the Social Force Model. In IEEE Conference on Decision and Control (CDC), 2013. Submitted.

[4] E. T. Hall. Proxemics - the study of man's spatial relations. In Man's image in medicine and anthropology. International Universities Press, 1962.

[5] D. Helbing, I. Farkas, and T. Vicsek. Simulating dynamical features of escape panic. Nature, 407:487-490, September 2000.

[6] D. Helbing, I. J. Farkas, and T. Vicsek. Freezing by heating in a driven mesoscopic system. Phys. Rev. Lett., 84:12401243, 2000.

[7] D. Helbing and P. Molnár. Social force model for pedestrian dynamics. Phys. Rev. E, 51:4282-4286, 1995.

[8] M. Jarke, X. T. Bui, and J. M. Carroll. Scenario management: An interdisciplinary approach. Requirements Engineering, 3(3-4):155-173, 1998.

[9] A. Johansson, D. Helbing, and P. K. Shukla. Specification of the social force pedestrian model by evolutionary adjustment to video tracking data. Advances in Complex Systems, 10(supp02):271-288, 2007.

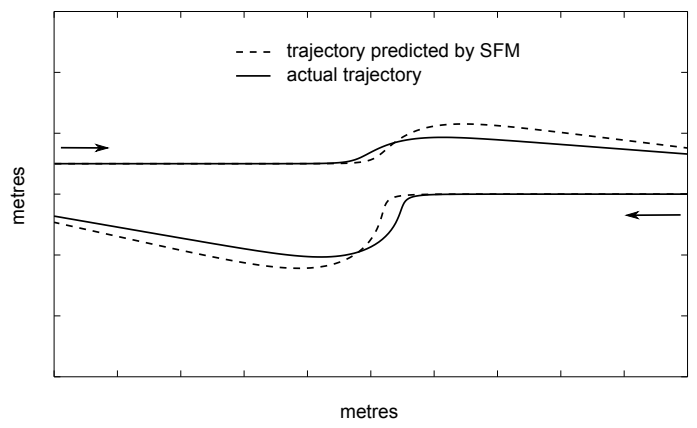

(a) Good prediction of smooth flow in opposite directions.

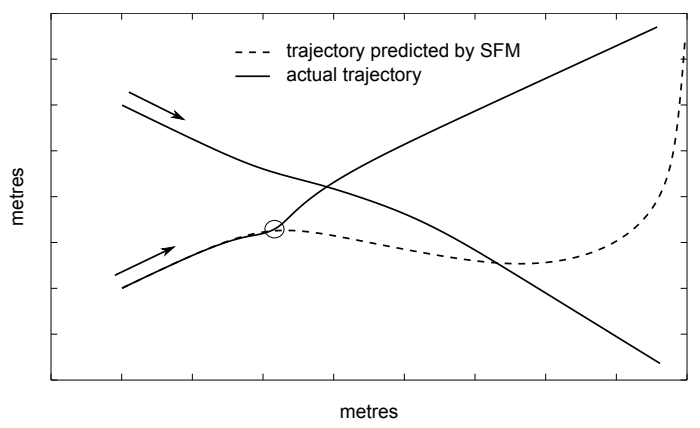

(b) Poor prediction of crossing paths.

Figure 6. Performance of parametrised SFM.

[10] T. I. Lakoba, D. J. Kaup, and N. M. Finkelstein. Modifications of the helbing-molnar-farkas-vicsek social force model for pedestrian evolution. Simulation, 81(5):339$352,2005$.

[11] M. Luber, J. A. Stork, G. D. Tipaldi, and K. O. Arras. People tracking with human motion predictions from social forces. In Proc. IEEE International Conference on Robotics and Automation (ICRA'10), Anchorage, USA, 2010.

[12] Microsoft Corp. Redmond WA. Kinect for Xbox 360. http://www.xbox.com/en-US/Kinect.

[13] D. R. Parisi, M. Gilman, and H. Moldovan. A modification of the social force model can reproduce experimental data of pedestrian flows in normal conditions. Physica A: Statistical Mechanics and its Applications, 388(17):3600 3608, 2009.

[14] W. B. Rouse and N. M. Morris. On looking into the black box: Prospects and limits in the search for mental models. Psychological Bulletin, 100(3):349, 1986.

[15] R. Teague, K. De Jesus, and M. N. Ueno. Concurrent vs. post-task usability test ratings. In Proceedings of Computer-Human Interaction (CHI'01), pages 289-290. ACM Press, 2001.

[16] S. Wei-Guo, Y. Yan-Fei, W. Bing-Hong, and F. WeiCheng. Evacuation behaviors at exit in CA model with force essentials: A comparison with social force model. Physica A: Statistical Mechanics and its Applications, 371(2):658-666, 2006.

[17] P. Wittenburg, H. Brugman, A. Russel, A. Klassmann, and H. Sloetjes. Elan: a professional framework for multimodality research. In Proceedings of LREC, volume 2006, 2006.

[18] W. J. Yu, R. Chen, L. Y. Dong, and S. Q. Dai. Centrifugal force model for pedestrian dynamics. Phys. Rev. E, 72:026112, Aug 2005. 